\title{
Real-Time Dense Stereo Matching with ELAS on FPGA Accelerated Embedded Devices
}

\author{
Oscar Rahnama ${ }^{1,2}$, Duncan Frost ${ }^{1}$, Ondrej Miksik ${ }^{1,3}$ and Philip H.S. Torr ${ }^{1}$
}

\begin{abstract}
For many applications in low-power real-time robotics, stereo cameras are the sensors of choice for depth perception as they are typically cheaper and more versatile than their active counterparts. Their biggest drawback, however, is that they do not directly sense depth maps; instead, these must be estimated through data-intensive processes. Therefore, appropriate algorithm selection plays an important role in achieving the desired performance characteristics.

Motivated by applications in space and mobile robotics, we implement and evaluate an FPGA-accelerated adaptation of the ELAS algorithm. Despite offering one of the best trade-offs between efficiency and accuracy, ELAS has only been shown to run at $1.5-3$ fps on a high-end CPU. Our system preserves all intriguing properties of the original algorithm, such as the slanted plane priors, but can achieve a frame rate of $47 \mathrm{fps}$ whilst consuming under $4 \mathrm{~W}$ of power. Unlike previous FPGA based designs, we take advantage of both components on the CPU/FPGA System-on-Chip to showcase the strategy necessary to accelerate more complex and computationally diverse algorithms for such low power, real-time systems.
\end{abstract}

Index Terms-Range Sensing, RGB-D Perception

\section{INTRODUCTION}

$\mathbf{I}$ $\mathrm{N}$ many areas of robotics, such as autonomous navigation [1], [2], [3] and manipulation/grasping [4], not only is the ability to perceive depth critical, but it needs to be obtained very accurately and in real-time. On mobile or embedded platforms, power consumption, cost, size and weight also become important factors to consider. For instance, assistive augmented glasses should be mobile, lightweight and ergonomic whilst retaining the ability to operate for long periods on limited battery power [5], [6].

Active methods of measuring depth, which are commonly used due to their high accuracy, carry certain disadvantages. LIDAR systems are often bulky, heavy and costly. Infrared systems, on the other hand, are limited in their range, susceptible to interference and, more importantly, constrained by ambient lighting. Passive methods may not be limited by these factors, however, they are computationally very expensive and their accuracy/latency depends heavily on the techniques used.

Manuscript received: September, 10, 2017; Revised December, 7, 2017; Accepted January, 12, 2018.

This paper was recommended for publication by Editor Cyrill Stachniss upon evaluation of the Associate Editor and Reviewers' comments. This work was supported by People Programme (Marie Curie Actions - "Initial Training Networks") of the EU FP7 under REA grant No. 317497 (EDISON), Technicolor, ERC grant ERC-2012-AdG 321162-HELIOS, EPSRC grant Seebibyte EP/M013774/1, EPSRC/MURI grant EP/N019474/1.

${ }^{1}$ Department of Engineering, University of Oxford, UK

Oscar.Rahnama@eng.ox.ac.uk

${ }^{2}$ OxSight Ltd ${ }^{3}$ Emotech Labs

Digital Object Identifier (DOI): see top of this page.
Stereo matching algorithms can be broadly split into global energy minimization methods and local matching techniques. The former are often more accurate, but the generally large/irregular memory requirements and sequential/iterative nature of their underlying algorithms make them dependent on powerful processors for speed up, and even then, their frame rate is limited. Conversely, local methods struggle with textureless and occluded regions, but the uniformity of their computations and the absence of dependencies between pixels makes them very suitable parallel acceleration.

Benefiting in part from the greater accessibility provided by CUDA/OpenCL, such acceleration has been predominantly done with Graphics Processing Units (GPU). However, Field Programmable Gate Arrays (FPGA) are becoming increasingly competitive alternatives, especially in power limited systems, with their capacity for in-stream processing, adherence to strict timings and supremacy at sliding-window operations [7].

Their effectiveness for stereo image processing has been previously demonstrated [8], [9], [10] with the most accurate implementations usually relying on Semi-Global Matching (SGM) [11]. However, as SGM is highly recursive, memory intense and, in its entirety, ill suited to acceleration, those that do either only partially implement it or sacrifice latency and throughput by relying heavily on external memory.

In this paper, we investigate the adoption and acceleration of a competing algorithm for low-power embedded systems. The algorithm, Efficient Large-Scale Stereo (ELAS) [12], is the fastest CPU algorithm w.r.t. resolution on the Middlebury dataset [13] and one of the most accurate non-global methods. ELAS is attractive since it very efficiently implements a slanted plane prior while its dense depth estimation is fully decomposable over all pixels and, hence, suitable for parallel processing. Unfortunately, the intermediate step, i.e. estimation of coarse scene geometry through the triangulation of support points, is a very iterative, sequential and conditional process with an unpredictable memory access pattern; making it difficult to accelerate on an FPGA.

To overcome this challenge, we propose the first stereo implementation which collaboratively utilizes both components of an embedded CPU-FPGA System on Chip (SoC) for the purpose of algorithm acceleration ${ }^{1}$. Other published low-power systems achieve good frame rates by limiting the algorithms they implement to those that can be fully processed by the FPGA, even when closely coupled processors are available e.g. [2], [1]. We, instead, seek to take advantage of both available components to efficiently accelerate the more

\footnotetext{
${ }^{1}$ Source code available at https://github.com/torrvision/ELAS_SoC
} 


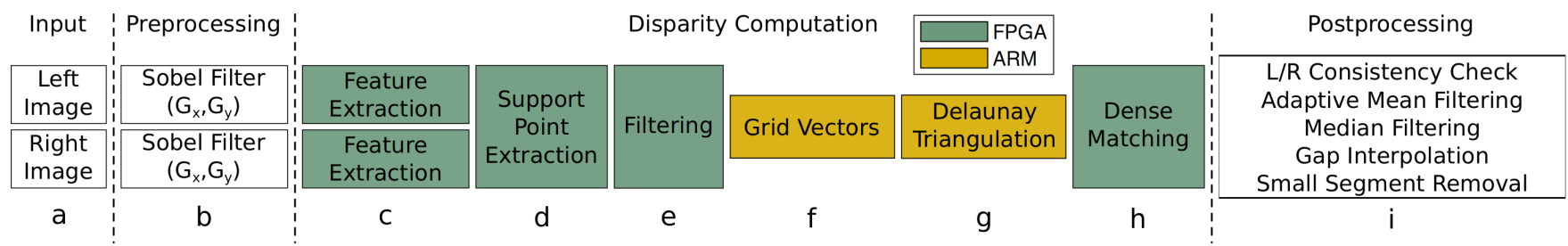

Fig. 1. ELAS Overview: Extract a set of support points from gradient images that are then used to establish priors for the dense matching stage.

complicated/accurate ELAS algorithm and demonstrate its feasibility for low-power systems. Accomplishing this involves offloading the different stages of the processing pipeline onto the component that best suits the computations involved. We discuss the rational behind the chosen partitioning and explain why its the most suitable, describe the key traits required in the design of efficient accelerators as well as the changes made to best adapt the algorithm to the platform. Tested on both the KITTI and New Tsukuba data sets, our system outperforms the frame rate of the original by $\sim 15-30 \times$ with a rate of 47 fps ( $1242 \times 375$ images) and, in addition, improves upon its accuracy - all the while with under $4 \mathrm{~W}$ of power consumption.

\section{RELATED WORK}

The pursuit of real-time stereo began in the 1980's [14]. Initial implementations of dense stereo minimized relatively simple matching costs, e.g. Sum of Absolute Differences (SAD) or Sum of Squared Differences (SSD), between left and right image patches evaluated along the epipolar lines. Kayaalp and Eckman [15] were one of the first to present such a system, capable of estimating disparity over a 64 disparity range in about one second for $256 \times 256$ images.

The first system capable of at least $30 \mathrm{fps}-$ on $200 \times 200$ images with a 23 pixel disparity range - was on a custom platform built from off-the-shelf components by Kanade $e t$ al. [16], [17]. Similarly to [15], they used a Sum of Sum of Absolute Difference (SSAD) but rather than summing over the different color channels, they summed over the six different cameras of their multi-camera system.

The first FPGA implementation of dense stereo matching used 16 Xilinx 4025 FPGAs [18]. It relied on the Census Transform (CT) [19] and computed 24 disparity levels over $320 \times 240$ images at $42 \mathrm{fps}$. Over about the next decade, FPGAs were repeatedly demonstrated as suitable platforms for dense real-time stereo, however they mostly implemented only variations of the SAD, SSAD, zero-mean SAD (ZSAD) and $\mathrm{CT}$ with additional noise suppressing post-processing steps [20], [21], [22], [23], [24]. Hence, the accuracy of such approaches was not typically comparable with state-of-the-art models which were formulated in global energy minimization frameworks [13], [25], [26].

Notable improvements in accuracy of FPGA implementations were made by incorporating Semi-Global Matching (SGM) [11]. For instance, Gehrig et al. [27] used a $3 \times 3$ window ZSAD along a 64 disparity range and minimized over 8 separate directions to run at $27 \mathrm{fps}$. Banz et al. [8] proposed a similar solution, but only aggregated costs over 4 directions with a rank transform [19]. SGM's larger scope managed to partly bridge the accuracy gap between strictly local operators and global optimization methods whilst remaining suitable for acceleration. However, SGM still has disadvantages such as large memory requirements and a fronto-parallel bias. Alternative recent approaches have shown some improvements in both frame rate and accuracy [28], [29], [30], [31], [9], [10], [32], however, they still lack the accuracy of SGM.

During the same period (2000-2010), graphics processing units (GPU) began to appear as alternative platforms for algorithm acceleration [33], [34], [35]. Although they offered speedup for sliding window algorithms such as local stereo, GPUs typically under-performed and consumed more power than their FPGA counterparts [7]. They were therefore a less favorable option for truly embedded and real-time systems.

Currently, the fastest CPU stereo algorithm on the Middlebury dataset [13], normalized w.r.t. resolution, is Efficient Large-Area Stereo [12]. It competes with SGM in accuracy, but its diverse computational nature has it overlooked in favor of other fully FPGA implementable algorithms. With new closely coupled CPU/FPGA System-on-Chip devices, however, it stands to benefit a lot in terms of acceleration.

\section{PReliminaries}

\section{A. Original ELAS algorithm}

The Efficient Large-Scale Stereo Matching (ELAS) algorithm [12] relies on the assumption that not all correspondences are equally difficult. It first establishes a set of sparse correspondences whose estimation is simpler and at the same time comes with a higher degree of confidence. These correspondences provide a coarse approximation of the scene geometry and are used to define a slanted plane prior which guides the dense matching stage.

An overview of the ELAS is shown in Fig. 1. To obtain the set of sparse but confident correspondences (the "support points"), the stereo pair first passes through a SAD matching stage over the horizontal and vertical gradients of the images, Fig. 1(b-d). The resulting set is sparse as only pixels with sufficiently unambiguous disparity values are kept. This criterion is measured by comparing the distance between the first and second minima of the SAD evaluations across the disparity range. The results from this stage then undergo a further filtering procedure, Fig. 1(e), to remove implausible and redundant values which would respectively corrupt or unnecessarily complicate the coarse 3D representation. This filtering process compares the sparse values to neighbors within a window region to ensure that they are consistent and removes identical values along the same row or column.

The set of support points is then used to guide the dense stereo matching stage (Fig. 1(h)) in two separate ways. First, 


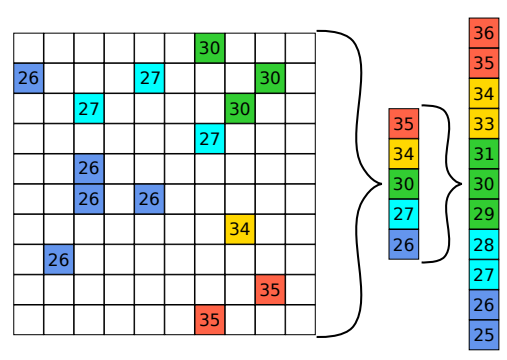

Fig. 2. Pooling support points within a sub-region to create a grid vector. The sparse set of correspondences in every given grid-region of an image are pooled together to create a characteristic search vector. This search vector is expanded to include immediate neighbors of included support points $( \pm 1)$

the set of support points is used to define a slanted plane prior which guides the dense matching stage. To this end, ELAS uses Delaunay triangulation to construct a mesh which approximates coarse scene geometry (Fig. 1(g)). Second, this slanted plane prior is used to limit the disparity values evaluated during the dense matching stage. This range is expanded to include immediately neighboring values $( \pm 1)$, which gives an algorithm a chance to recover in case the initial mesh is incorrect (Fig. 2 and Fig. 1(f)).

Following the dense matching stage, ELAS uses postprocessing (Fig. 1(i)) to invalidate occluded pixels and further improves smoothness across the image. Although postprocessing plays an important role in obtaining an accurate final result, it is not a core part of the algorithm or unique to it, and therefore ignored.

\section{B. Platform}

We use Xilinx's ZC706 development board with the $\mathrm{XC7Z045} \mathrm{SoC;} \mathrm{this} \mathrm{is} \mathrm{a} \mathrm{heterogeneous} \mathrm{chip} \mathrm{that} \mathrm{incorporates}$ an ARM Cortex A9 processor operating at $800 \mathrm{MHz}$ and a $28 \mathrm{~nm}$ Kintex series FPGA on the same die. The collocation of the two components ultimately serves to increase the overall throughput of the system as it allows for rapid and efficient exchange of data. The resources available on the FPGA include: 218600 Look Up Tables (LUT), 437200 Flip Flops (FF), 900 DSP48 Blocks, 1090 18K Block RAMs (BRAM).

\section{High level synthesis}

Vivado High Level Synthesis (VHLS) provides a higher abstraction approach to FPGA block implementation by synthesizing designs described in a high level language such a $\mathrm{C} / \mathrm{C}++$ into equivalent hardware descriptions. A deep understanding of the underlying hardware architecture is still required, but it alleviates the burden of adopting a low level hardware description languages such as VHLD/Verilog. By abstracting fine grained, less critical details of the design, VHLS accelerates and facilitates development with FPGAs. Although accelerators designed with this higher abstraction approach may not be as optimized or as resource efficient as those designed with low level hardware description languages, the ability to deploy, modify and test them much more rapidly is a reasonable compromise. Hence, we implemented all accelerators using VHLS.

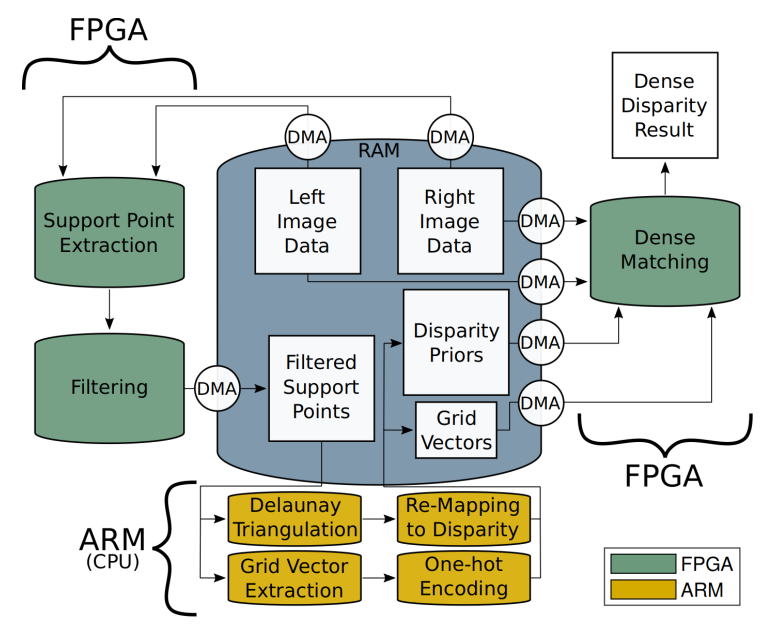

Fig. 3. Overview of System-on-Chip (SoC) implementation. Computeintensive tasks are offloaded to FPGA accelerators whereas conditional/sequential tasks are handled by ARM CPU. Communication between CPU and FPGA is handled by Direct Memory Access blocks in the FPGA.

\section{SySTEM OVERVIEW}

Fig. 3 shows the overall system implemented on Zynq SoC platform. Determining which parts of the algorithm are offloaded onto dedicated accelerators and which are proceed on the ARM CPU is a twofold process. First, the entire algorithm's CPU runtime is profiled to get an estimate of the time spent in each function. The main bottlenecks are then identified and the algorithm is mapped and broken down into its main components. In the subsequent step, the computational nature of the different components are evaluated and matched to the most appropriate component for processing.

a) FPGA: FPGA accelerators are severely hampered if they require communicating with external memory or if they contain many divergent datapaths through them. However, they excel at performing a variety of operations, simultaneously, on a large range of data. As such, functions that process blocks of data with well defined, relatively local, memory access patterns and limited amounts of conditional branching can benefit tremendously from such acceleration.

In ELAS, the functions responsible for support point extraction, filtering and block matching fit such criteria. Therefore, as denoted by the green blocks in Fig. 3, these are offloaded onto dedicated FPGA accelerators. These accelerators can either have data transferred in-between them directly (Sparse $\rightarrow$ Filtering) or to and from the the RAM through Direct Memory Access (DMA) blocks in the FPGA (programmed by the $\mathrm{CPU})$. The accelerators process the data in-stream only storing small portions of the overall stream ( $c f$., Sec. V) and outputting data at the same rate at which it is received. This processing style best compliments the raster pixel readout of modern image sensors and allows for top level pipelining in between successive FPGA accelerators.

b) ARM CPU: Functions with very unpredictable memory access patterns as well as those with a high amount of conditional branching are very ill suited for FPGA acceleration. These, instead, benefit more from the ARM CPU's faster processing speeds, its sequential processing style (invariant 


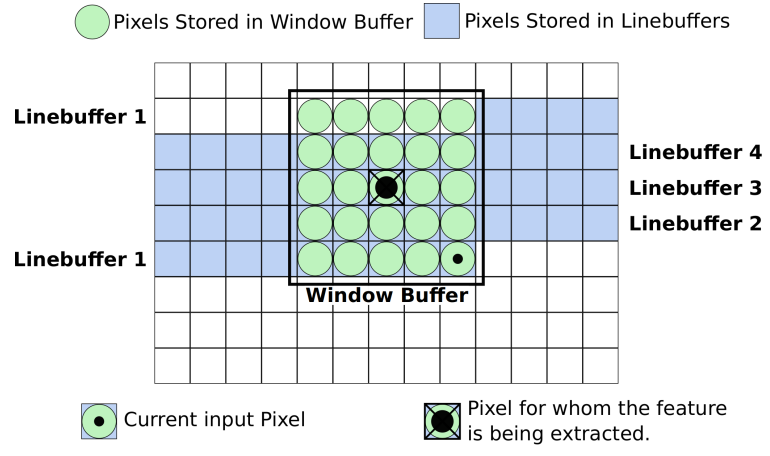

Fig. 4. Memory requirements for sliding window operations in FPGA accelerators. Line buffers (blue) are used to store large amounts of data but can used to provide a single value per clock cycle. Window buffers (green) are local registers used to store immediately required data.

to branching) and its equal, but longer, access to memory (disregarding cache hit/misses).

As denoted by the yellow blocks in Fig. 3, the ELAS processes that are handled in this manner are the Delaunay triangulation as well as the remapping of the slanted plane priors into disparity priors. Grid vector extraction and one-hot encoding ( $c f$., Sec. VI) are also done on the ARM. Although grid vector extraction would appear to be a candidate for FPGA acceleration as it "pools" values within a local memory region with a window like operation, as shown in Fig. 2, in reality, as it only operates on a single value at a time, it benefits more from the faster clock of ARM.

\section{Key Accelerator Design Traits}

\section{A. FPGA memory management}

To achieve the required parallelism within the FPGA blocks, all the necessary data for a set of computations must be available on the same clock cycle as those computations are to occur. Memory management, therefore, has the largest impact on accelerator throughput and latency. Fig. 4 shows the combination of block RAMs and local memory that are used as the core components for this purpose. Block RAMs (BRAMs) are the most resource efficient stores of large quantities of data. In the accelerators, they behave as line buffers, storing previously received pixel information into the FPGA fabric. For a given $W \times W$ matching window, each image's pixel data is collected by a set of $2 \times W$ line buffers. On every clock cycle, each line buffer shifts its contents at a given index to the line buffer above it and a new pixel is read into the free space created at that index of the bottom-most buffer. Similarly, the data that was in the topmost buffer is shifted out as it is no longer required - only a fraction of the overall image data is ever held in the FPGA.

Each BRAM, however, is only able to read and write one value per clock cycle. Therefore, a set of line buffers only supplies one column of pixel data per clock cycle. Most computations in the accelerators, however, operate over windows of pixels and therefore this alone is insufficient in meeting the memory requirements for a high throughput/low latency design. Instead, an additional $W \times W$ size "window" buffer is necessary (Fig. 4). As it consists of local registers within the
FPGA block which are all instantaneously accessible, this is resource expensive. On every clock cycle, the contents of the window are updated by shifting all columns once to the left. The rightmost column is read in from the values stored in the line buffers (including the latest pixel value).

By combining the use of storage elements in this manner, we efficiently achieve access to all the necessary data on the same clock cycle on which it is used. No additional clock cycles need to be spent on memory access.

\section{B. Pipelining}

Although VHLS handles timing considerations and data flow control of FPGA accelerators, the throughput and latency it achieves depends on the propagation delays within the accelerators as well as the desired amount of pipelining and overall clock frequency.

When maximizing the throughput of an accelerator, pipelining is necessary when its total internal propagation delay exceeds the clock period to which it is constrained (pixel read in/out rate). By introducing pipelining, the accelerator is able to meet the clock frequency constraint by dividing and spreading its operation over multiple clock cycles. Each sub-stage is separated from the next with flip flops that store intermediate values and therefore pipelining improves utilization. Ultimately, however, it results in the accelerator being shared across a set of inputs as each sub-stage processes a new input on every clock cycle - a larger amount of data is being simultaneously acted upon.

Other than the flip flop requirement, the trade-off with pipelining is that the number of clock cycles between first input and output increases by the amount of pipeline stages and the propagation delay experienced by a single pixel is longer as each sub-stage's delay is extended to that of the longest sub-stage. In image processing, however, as large quantities of pixel data pass through the accelerators, the latency introduced by pipelining is not only negligible, but significantly outweighed by the ability to output data at a much faster rate. It plays a significant role in achieving the desired frame rate in our design.

\section{Platform Conscious Algorithm Changes}

\section{A. Feature selection}

Efficient implementation of original ELAS uses SIMD accelerators with fixed widths of 16 bytes for feature extraction and matching. Such an implementation, however, lacks flexibility since the number of pixels it can process is limited and must be in multiples of 16 (for 8 bits). The result is that for a given $W \times W$ window, only a subset of pixels contained within it are used for matching purposes.

Due to our memory management, all pixel data within a window is available and therefore no speed penalty is incurred by using it (Fig 4). It also improves the accuracy since we use a larger number of pixels in the matching process. We use the Census Transform descriptors with Hamming Distance instead of the SAD as it achieves illumination invariant matching without the need for an additional pre-processing step (Sobel filter Fig. 1(b)). 


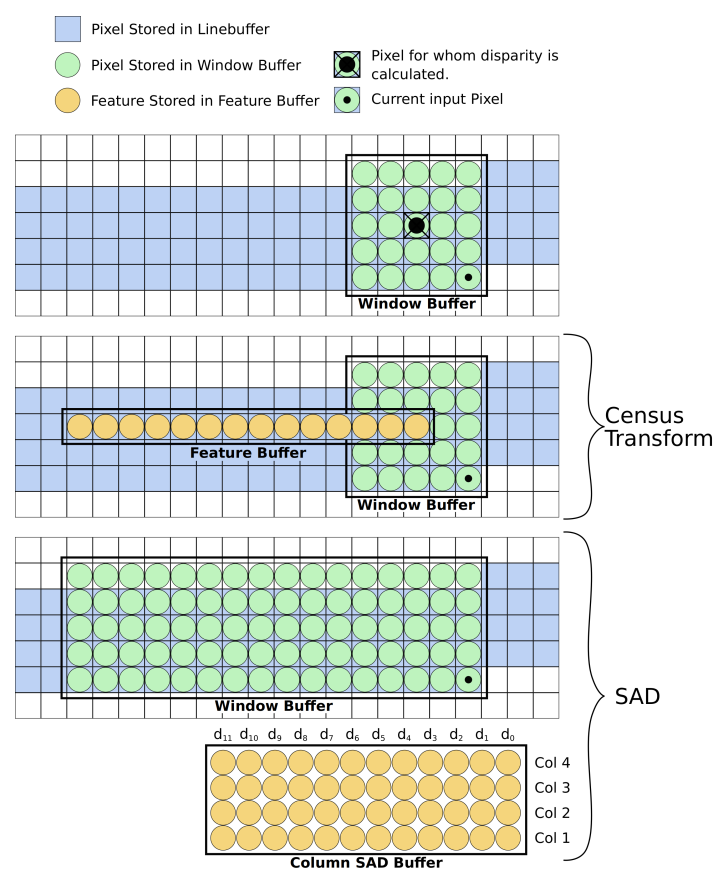

Fig. 5. Comparison of Extraction and Matching Implementation with Census Transform and Sum of Absolute Differences

Even if the pre-processing is discounted, the SAD generally requires more resources than the CT. As shown in Fig. 5, unlike the CT that reuses previously extracted features, the SADs must be recomputed every time. Also, SAD implementations that achieve similar throughput, such as the one in [36], require an additional window buffer to store previous column SAD computations (bottom of Fig. 5). Thus the resource requirement is greater due to both the greater number of computations as well as the greater need for memory.

These changes result in a feature descriptor that is shorter in bit length $(81 / 25$ compared to the original $512 / 128)$, while containing information about larger pixel neighborhood than ELAS's CPU implementation.

\section{B. Measuring ambiguity}

The support point extraction is done slightly differently to the original algorithm. We replace the original criteria which assumes a match is unambiguous if

$$
\frac{m_{1}}{m_{2}} \leq 0.9
$$

where $m_{1}$ and $m_{2}$ are the first and second mimima respectively. This is equivalent to thresholding $m_{1}$

$$
m_{1} \leq 0.9 m_{2}=T_{\text {err }}\left(m_{2}\right),
$$

However, implementing such comparison on an FPGA requires a number of DSP blocks. Hence we approximate the threshold $T_{e r r}\left(m_{2}\right)$ with a shift-sum

$$
T_{\text {err }}\left(m_{2}\right)=0.9 m_{2} \approx \frac{m_{2}}{2}+\frac{m_{2}}{4}+\frac{m_{2}}{8}+\frac{m_{2}}{32}=0.90625 m_{2} .
$$

This eliminates the need for DSP blocks as shift-summing is fully accomplished within the LUT fabric of the FPGA.
(A)

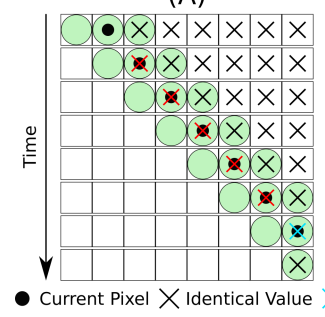

(B)

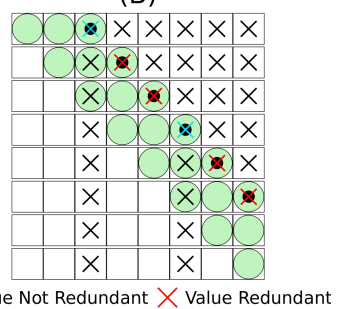

Fig. 6. One dimensional simplification of redundancy verification (A) Searching both forwards and backwards propagates redundancy into the value's nonexistence (B) Searching strictly backwards retains the value at the desired frequency

\section{Filtering support points}

The original algorithm uses both past and future values in the data stream for redundancy check. As shown in the simplified 1-D example of Fig. 6(A), the shared values used to flag redundancy are often made redundant by instances further ahead. Instead, the "filter" FPGA accelerator only relies on past values when determining redundancy. This ensures the shared values are less frequent rather than non-existent.

\section{Data Reduction}

To accommodate for the static bit-width of accelerator ports and to minimize the data transferred to the FPGA, we introduce new data reduction steps to ELAS (on the ARM). Referring to Fig. 3, the first one-hot encodes the grid vectors from a variable length byte array into a statically sized bitwise representation. The second converts the result of the Delaunay Triangulation from a variable mesh of triangles into a static, input image sized, matrix of disparity priors.

\section{EXPERIMENTAL RESULTS}

To demonstrate the effectiveness of our approach, we provide a detailed evaluation across differently parameterized sets of implementations and evaluate them on both the KITTI and New Tsukuba data sets.

As FPGA accelerators can not be easily reconfigured for different image resolutions during testing, we only use 310 of the 400 KITTI image pairs that have the same $1242 \times 375$ resolution. The New Tsukuba consists of 1800 images with resolution of $640 \times 480$, of which we use the provided subset of 200 image-pairs.

\section{A. Accuracy}

We begin by verifying how the accuracy of the FPGA accelerated version of ELAS, following the modifications made to adapt it to the SoC platform, compares to that of the original algorithm. To this end, we use the standard accuracy metric from the KITTI benchmark which measures the relative number of estimated disparities which differ from ground truth by both an absolute amount of at least 3 and a relative one of at least $5 \%$.

To ensure a fair comparison, the number of support points used to establish the prior should be approximately the same. As not all pixels are considered for support point extraction 
in the original algorithm, we find that this occurs when we use $\frac{1}{14}$ th of the number of total extracted support points in our method. We also use the same window sizes for both matching stages, i.e. $9 \times 9$ and $5 \times 5$, respectively. As previously explained, accuracy is measured without postprocessing/refinement as these processes aren't unique to ELAS and are more susceptible to dataset "fine tuning". With these parameters, the original implementation tested without post-processing over the same image set, obtains an average error of $17.9 \%$ while our implementation achieves an improved $16.5 \%$. With many other configurations tested, Table I, we find that the embedded version is generally more accurate, except when window sizes are made too small or when support points are overly sub-sampled. Unlike the hard-coded $9 \times 9$ and $5 \times 5$ windows of the original, we are able to quickly configure different window sizes to vary accuracy without any impact on frame-rate. As larger windows provide more information in matching, they also result in more accurate depth maps. However this only holds up to a certain size; eventually the inherent fronto-parallel bias of square matching windows begins negatively impact results.

\section{B. Per-frame processing time}

Fig. 7 (left axes) illustrates the proportion of the overall processing time of the ARM against that of the FPGA. The FPGA portion is inclusive of the time spent transferring data by the DMAs. Additionally, the results are reported across different support point densities which we regulate through down sampling. As shown, although the time spent on the ARM is proportional to the number of support points used and shortens significantly with down sampling, it nonetheless dominates the overall processing time.

In contrast, the combined processing time of the FPGA accelerators is mostly unaffected by changes in parameters such as matching window size, disparity range or the number of support points. As they have a constant throughput of 1 pixel/clock cycle, their processing time is, instead, predominantly a function image resolution. On average, it takes only $4.84 \pm .02 \mathrm{~ms}$ for the KITTI images $(1242 \times 375 \mathrm{px})$ and $3.19 \pm .02 \mathrm{~ms}$ on the New Tsukuba $(640 \times 480 \mathrm{px})$. The $1.51 \times$ difference corresponds exactly to the pixel ratio difference.

The line plot in Fig. 7 (right axes) shows the error percentage vs. the number of support points controlled by downsampling. Whilst slightly unintuitive, the best accuracy is not achieved with the largest number of support points (peak at $\frac{1}{8}$ of the support points). This is likely due to the noisy nature of sparse correspondence matching. Following this peak, accuracy gradually decreases with reduced number of support points as the resulting planar surfaces become less accurate coarse approximations of scene geometry.

Interestingly, although the FPGA accelerators run at constant time, the matching window size of the support point extraction stage is negatively correlated with overall frame rate. Larger windows do incur a greater initial latency to account for the additionally used line buffers, but this difference is negligible (evidenced by invariance of the frame rate to the window size of dense matching) and can not account for

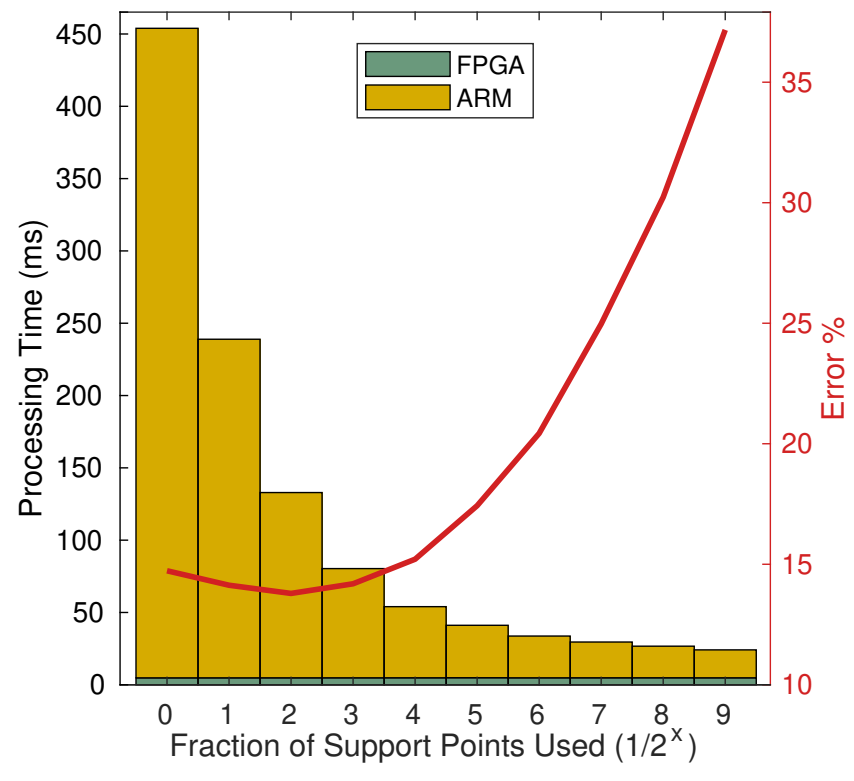

Fig. 7. Processing time and related accuracy w.r.t down sampling

this difference. Instead, the frame rate reduction is actually due to the increased number of support points resulting from the extraction using larger matching windows - this impacts the ARM's workload. Therefore, the main bottleneck is the Delaunay Triangulation which is in stark contrast to the one reported in the original CPU implementation.

\section{Power and resource consumption}

The high throughput capability of accelerators is limited by the number of circuit elements available within the FPGA fabric. In order to report this "resource utilization" (Table I), we split it into the three main types of blocks (we exclude DSP blocks as they are negligibly used). As expected, the LUTs, used for the combinational logic and instantaneous memory, are the most predominantly utilized and this amount depends strongly on the window size of dense matching. The flip flops, utilized primarily for pipelining, share a similar dependency, but to a lesser extent. The BRAMs, used as line buffers are purely a function of the cumulative image rows required for a given set of windows.

One of the most important advantages of the proposed implementation is the power efficient computing that it enables ( $c f$. ., last two columns of Table I). The ARM processor, running at a steady $800 \mathrm{Mhz}$, accounts for a constant but majority share of the power. In contrast, the power consumed by the FPGA is much more controlled and directly proportional to the portion of FPGA logic that is being utilized. Altogether, however, the results highlight that the implementation is not only capable of running the algorithm in real-time, it succeeds in doing this with under $3 \mathrm{~W}$ of power (in contrast to powerful desktop CPUs which typically require $>100 \mathrm{~W}$ ).

\section{Throughput}

To further increase frame rate, we explore operating over multiple images simultaneously by taking advantage of the 
TABLE I

IMPACT OF WINDOW SIZES USED DURING MATCHING ON FRAME RATE, ACCURACY AND RESOURCE UTILIZATION

\begin{tabular}{|c|c|c|c|c|c|c|c|c|c|c|c|c|c|}
\hline \multirow{2}{*}{ Data Set } & \multirow{2}{*}{$\begin{array}{c}\text { Window } \\
\text { Size }\end{array}$} & \multirow{2}{*}{$\begin{array}{c}\text { Window } \\
\text { Size }\end{array}$} & \multicolumn{2}{|c|}{ CPU ELAS } & \multicolumn{2}{|c|}{$1 / 8$} & \multicolumn{2}{|c|}{$1 / 32$} & \multicolumn{3}{|c|}{ Resource Utilization [\%] } & \multicolumn{2}{|c|}{ Power (Watts) } \\
\hline & & & FPS & Error \% & FPS & Error \% & FPS & Error \% & LUT & FF & BRAM & ARM & FPGA \\
\hline \multirow{12}{*}{$\begin{array}{c}\text { KITTI } \\
1242 \times 375\end{array}$} & \multirow{3}{*}{$7 \times 7$} & $3 \times 3$ & \multirow{12}{*}{$1.5-3$} & \multirow{12}{*}{17.9} & 17.3 & 18.4 & 29.2 & 23.2 & 22.0 & 14.5 & 10.5 & 1.70 & 0.91 \\
\hline & & $5 \times 5$ & & & 17.4 & 16.5 & 29.2 & 21.7 & 24.2 & 15.3 & 10.8 & 1.70 & 1.06 \\
\hline & & $7 \times 7$ & & & 17.4 & 15.8 & 29.2 & 21.4 & 27.2 & 16.7 & 11.2 & 1.70 & 1.08 \\
\hline & \multirow{3}{*}{$9 \times 9$} & $3 \times 3$ & & & 12.5 & 17.2 & 24.5 & 20.0 & 26.2 & 17.1 & 10.8 & 1.70 & 1.00 \\
\hline & & $5 \times 5$ & & & 12.4 & 14.5 & 24.5 & 17.7 & 28.5 & 17.9 & 11.2 & 1.70 & 1.17 \\
\hline & & $7 \times 7$ & & & 12.3 & 13.7 & 24.3 & 17.0 & 31.7 & 19.3 & 11.6 & 1.70 & 1.16 \\
\hline & \multirow{3}{*}{$11 \times 11$} & $3 \times 3$ & & & 10.5 & 17.6 & 22.3 & 19.5 & 32.0 & 20.4 & 11.2 & 1.70 & 1.08 \\
\hline & & $5 \times 5$ & & & 10.5 & 14.5 & 22.3 & 16.6 & 34.0 & 21.2 & 11.6 & 1.70 & 1.20 \\
\hline & & $7 \times 7$ & & & 10.5 & 13.6 & 22.3 & 15.7 & 37.3 & 22.6 & 11.9 & 1.70 & 1.21 \\
\hline & \multirow{3}{*}{$13 \times 13$} & $3 \times 3$ & & & 9.4 & 18.2 & 21.0 & 19.4 & 38.5 & 24.2 & 11.6 & 1.70 & 1.12 \\
\hline & & $5 \times 5$ & & & 9.5 & 14.9 & 21.0 & 16.3 & 40.4 & 25.1 & 11.9 & 1.70 & 1.27 \\
\hline & & $7 \times 7$ & & & 9.5 & 13.9 & 21.0 & 15.4 & 43.5 & 26.5 & 12.3 & 1.70 & 1.28 \\
\hline \multirow{2}{*}{$\begin{array}{c}\text { Tsukuba } \\
640 \times 480 \\
\end{array}$} & $9 \times 9$ & $5 \times 5$ & \multirow{2}{*}{ N/A } & \multirow{2}{*}{6.4} & 17.6 & 6.8 & 36.2 & 6.4 & 28.1 & 17.0 & 9.7 & 1.70 & 1.00 \\
\hline & $11 \times 11$ & $7 \times 7$ & & & 14.8 & 6.4 & 32.9 & 5.9 & 37.4 & 21.7 & 10.5 & 1.70 & 1.23 \\
\hline
\end{tabular}

TABLE II

TIME BREAKDOWNS

\begin{tabular}{|c|c|c|c|}
\hline Cones $(900 \times 750)$ & $\begin{array}{c}\text { Time (ms) } \\
\text { i7-only (orig.) }\end{array}$ & $\begin{array}{c}\text { Time (ms) } \\
\text { ARM+FPGA }\end{array}$ & $\begin{array}{c}\text { Time }(\mathrm{ms}) \\
\text { i7+FPGA }\end{array}$ \\
\hline Support Points & 118 & 3.5 & 3.5 \\
\hline Triangulation & 7 & 84.42 & 7 \\
\hline Matching & 359 & 3.5 & 3.5 \\
\hline
\end{tabular}

additional core of the ARM and separate but identical accelerators in the FPGA. This effectively doubles the system and therefore the resources and power used by the FPGA double (minus some shared overhead). Conversely, the ARM's power consumption remains the same, at $1.7 \mathrm{~W}$. For example, a dual system with $(9 \times 9)$ and $(5 \times 5)$ matching windows utilizes $56.8 \%$ of LUTs, $35 \%$ of FFs, $22.8 \%$ of BRAMs and consumes $3.67 \mathrm{~W}$. The most accurate configuration using this multi-threaded/multi-accelerator approach runs at 23.7fps $(3.74 \mathrm{~W})$. A faster version, whose accuracy is poorer but still better than the original CPU versions runs at $47.0 \mathrm{fps}(3.67 \mathrm{~W})$.

\section{COMPARISON \& DISCUSSION}

Comparing these results to what was achieved in the original paper, it is clear that parallelizing key parts of the algorithm has successfully led to significantly faster - up to $30 \times$ real-time frame-rates. Despite the achievement, the results also reveal some of ELAS's weaknesses for power-limited platforms. From Table II, where the time breakdown for each stage is compared across systems, we see how the SoC manages $100 \times$ throughput increase for both matching stages even though it processes more data, i.e. all pixels considered for support point and full matching windows. However, with the low-power CPU paling in performance compared to its desktop counterpart, the triangulation procedure - seemingly insignificant in the original paper - is $>12$ times longer and dominates the processing time on the SoC. Thus, although ELAS is exemplified as one of the fastest stereo algorithm, its dependence on a very sequential procedure makes it also dependent on a powerful processor to achieve maximal speed up. In the last column of Table II, we show the processing times which one could obtain if an SoC, combining the same FPGA with an Intel Core i7 CPU instead of the ARM, were used to accelerate the algorithm with the same proposed approach. On such a platform, the frame rate of ELAS exceeds $70 \mathrm{fps}$, but it would also entail a power consumption in excess of 100 Watts.

In terms of accuracy, the improvements over the original can be attributed to the full matching windows/CT as opposed to the randomly sub-sampled SAD of the original. These subsampled windows were needed in the original to speed up the CPU processing time. In contrast, with the FPGA accelerators, not only is window matching speed independent of the number of pixels considered, but full windows result in a more efficient use of resources. As a corollary, unlike CPU ELAS whose runtime is coupled to its tailored windows, our embedded version can accommodate various window sizes and disparity ranges without being concerned about the impact on latency.

We compare the performance of our system to the fastest implementation currently reported on the KITTI benchmark, referred to as "CSCT+SGM+MF" (CSM) [37] and which, at its core, implements SGM - the competing algorithm in embedded, real-time systems. It reports an $8.24 \%$ error rate at $156 \mathrm{fps}$ on a $250 \mathrm{~W}$ NVIDIA Titan X. As CSM's result incorporates smoothing/refinement both inherently through SGM and through an additional median filter, we pass the results of our most accurate configuration through a median filter for the sake of comparison. With only this one additional post-processing step, we obtain a new error rate of 9.52\% already slightly better than the accuracy ELAS reports on the KITTI benchmark following all post-processing/refinement. Although CSM may still be marginally more accurate with a faster frame rate, it requires substantially more power at $250 \mathrm{~W}$. This is equivalent to a per Watt frame rate of $0.62 \mathrm{fps} / \mathrm{W}$. In their original paper, the authors also attempt a more power efficient implementation (same accuracy) on a mobile NVIDIA Tegra X1 GPU (10W). After scaling their results to the KITTI resolution, this more power efficient system manages only $13.8 \mathrm{fps}$ with a resulting efficiency of $1.38 \mathrm{fps} / \mathrm{W}$. Our system, in contrast, offers a $23.7 \mathrm{fps}$ frame rate with a corresponding $6.34 \mathrm{fps} / \mathrm{W}$ efficiency. This is a $10 \times$ improvement over the faster Titan X system and $5 \times$ over the slower Tegra X1 one.

The FPGA accelerators in this system were described in $\mathrm{C}++$ and then converted into logic with VHLS. In our experi- 
ence, although the tool did eliminate the need for writing lowlevel VHDL/Verilog, it still relied heavily on the user's deep knowledge of the target circuit. The original algorithm had to be completely re-engineered to comply with the hardware framework. As well as motivating the previously described modifications, this included adhering to a regular, timing-strict processing chain, minimizing any inter-process dependencies and eliminating conditional operations/branches/exceptions.

\section{CONCLUSION}

In this work, we have disassembled and reconstructed the ELAS algorithm onto an ARM + FPGA SoC with the purpose of evaluating its suitability for low-power, real-time embedded systems. By taking advantage of the immense parallelism available with FPGAs and by better adapting the algorithm for it, we not only successfully accelerate the frame rate by up to $30 \times$, but we have also demonstrate an improvement in accuracy. This is also entirely achieved with under $4 \mathrm{~W}$ of power, making 5-10 more efficient on a frame rate per Watt basis than competing algorithms on KITTI.

Through the iterative process of adapting the algorithm to the platform as well the starkly different resulting processing time breakdown we obtained, fundamental principles were gleaned for the future design of accurate, but ultimately realworld applicable, algorithms. Specifically, with parallelism being of paramount importance, any strictly sequential or iterative processes must be kept to a minimum as these will cause severe bottlenecks. Their acceleration depends on faster processors, and as CPU frequency is directly proportional to power consumption, this quickly incurs greater power requirements that are unrealistic in space, aerial or mobile robotics. Conversely, accelerators excel at simultaneously processing vast amounts of data as long as it is available and effectively managed in the fabric of the FPGA. Therefore, sacrificing accuracy by minimize the amount of pixels computed with has no impact on latency and should be entirely avoided.

Finally, although the newly developed high-level design tools by Xilinx do indeed facilitate the access, speed and and transportability of designing on FPGAs, one must be still possess a strong understanding of hardware design in order to efficiently implement accelerators with them.

\section{REFERENCES}

[1] H. Oleynikova, D. Honegger, and M. Pollefeys, "Reactive avoidance using embedded stereo vision for mav flight," in ICRA, 2015.

[2] G. Camellini, M. Felisa, P. Medici, P. Zani, F. Gregoretti, C. Passerone, and R. Passerone, "3DV-An embedded, dense stereovision-based depth mapping system," in Intelligent Vehicles Symposium Proceedings, 2014.

[3] N. A. Zainuddin, Y. M. Mustafah, Y. A. M. Shawgi, and N. K. A. M. Rashid, "Autonomous navigation of mobile robot using kinect sensor," in International Conference on Computer and Communication Engineering, 2014.

[4] C. Lehnert, I. Sa, C. McCool, B. Upcroft, and T. Perez, "Sweet pepper pose detection and grasping for automated crop harvesting," in ICRA, 2016.

[5] S. L. Hicks, I. Wilson, L. Muhammed, J. Worsfold, S. M. Downes, and C. Kennard, "A Depth-Based Head-Mounted Visual Display to Aid Navigation in Partially Sighted Individuals," PLOS ONE, 2013.

[6] O. Miksik, V. Vineet, M. Lidegaard, R. Prasaath, M. Nießner, S. Golodetz, S. L. Hicks, P. Perez, S. Izadi, and P. H. S. Torr, "The semantic paintbrush: Interactive $3 \mathrm{~d}$ mapping and recognition in large outdoor spaces," in ACM CHI, 2015.
[7] J. Fowers, G. Brown, P. Cooke, and G. Stitt, "A performance and energy comparison of fpgas, gpus, and multicores for sliding-window applications," in FPGA, 2012.

[8] C. Banz, S. Hesselbarth, H. Flatt, H. Blume, and P. Pirsch, "Real-time stereo vision system using semi-global matching disparity estimation: Architecture and fpga-implementation," in SAMOS, 2010.

[9] M. Pérez-Patricio, A. Aguilar-González, M. Arias-Estrada, H.-R Hernandez-de Leon, J.-L. Camas-Anzueto, and J. de Jesús OsunaCoutiño, "An fpga stereo matching unit based on fuzzy logic," Microprocessors and Microsystems, 2016.

[10] G. Cocorullo, P. Corsonello, F. Frustaci, and S. Perri, "An efficient hardware-oriented stereo matching algorithm," Microprocessors and Microsystems, 2016.

[11] H. Hirschmuller, "Accurate and efficient stereo processing by semiglobal matching and mutual information," in CVPR, 2005.

[12] A. Geiger, M. Roser, and R. Urtasun, "Efficient large-scale stereo matching," in ACCV, 2010.

[13] H. Hirschmuller and D. Scharstein, "Evaluation of cost functions for stereo matching," in CVPR, 2007.

[14] M. Drumheller and T. Poggio, "On parallel stereo," in ICRA, 1986.

[15] A. E. Kayaalp and J. L. Eckman, "Near real-time stereo range detection using a pipeline architecture," IEEE Transactions on Systems, Man, and Cybernetics, 1990.

[16] T. Kanade, H. Kano, S. Kimura, A. Yoshida, and K. Oda, "Development of a video-rate stereo machine," in IROS, 1995.

[17] T. Kanade, A. Yoshida, K. Oda, H. Kano, and M. Tanaka, "A stereo machine for video-rate dense depth mapping and its new applications," in $C V P R, 1996$.

[18] J. Woodfill and B. Von Herzen, "Real-time stereo vision on the parts reconfigurable computer," in FCCM, 1997.

[19] R. Zabih and J. Woodfill, "Non-parametric local transforms for computing visual correspondence," in ECCV, 1994.

[20] M. Hariyama, Y. Kobayashi, H. Sasaki, and M. Kameyama, "Fpga implementation of a stereo matching processor based on window-paralleland-pixel-parallel architecture," IEICE Transactions on Fundamentals of Electronics, Communications and Computer Sciences, 2005.

[21] S. Perri, D. Colonna, P. Zicari, and P. Corsonello, "Sad-based stereo matching circuit for fpgas," in Electronics, Circuits and Systems, 2006.

[22] C. Cuadrado, A. Zuloaga, J. L. Martin, J. Laizaro, and J. Jimenez, "Realtime stereo vision processing system in a fpga," in IECON, 2006.

[23] C. Georgoulas, L. Kotoulas, G. C. Sirakoulis, I. Andreadis, and A. Gasteratos, "Real-time disparity map computation module," Microprocessors and Microsystems, 2008.

[24] K. Ambrosch and W. Kubinger, "Accurate hardware-based stereo vision," CVIU, 2010.

[25] D. Scharstein and R. Szeliski, "A taxonomy and evaluation of dense two-frame stereo correspondence algorithms," IJCV, 2002.

[26] — , "High-accuracy stereo depth maps using structured light," in CVPR, 2003.

[27] S. K. Gehrig, F. Eberli, and T. Meyer, "A real-time low-power stereo vision engine using semi-global matching," in ICVS, 2009.

[28] C. Georgoulas and I. Andreadis, "A real-time fuzzy hardware structure for disparity map computation," Journal of Real-Time Image Processing, 2011.

[29] M. Werner, B. Stabernack, and C. Riechert, "Hardware implementation of a full hd real-time disparity estimation algorithm," IEEE Transactions on Consumer Electronics, 2014.

[30] W. Wang, J. Yan, N. Xu, Y. Wang, and F.-H. Hsu, "Real-time highquality stereo vision system in fpga," IEEE Transactions on Circuits and Systems for Video Technology, 2015.

[31] M. Pérez-Patricio and A. Aguilar-González, "Fpga implementation of an efficient similarity-based adaptive window algorithm for real-time stereo matching," Journal of Real-Time Image Processing, 2015.

[32] Y. Li, K. Huang, and L. Claesen, "Soc and fpga oriented high-quality stereo vision system," in FPL, 2016.

[33] M. Gong and Y.-H. Yang, "Near real-time reliable stereo matching using programmable graphics hardware," in CVPR, 2005.

[34] J. Lu, G. Lafruit, and F. Catthoor, "Fast variable center-biased windowing for high-speed stereo on programmable graphics hardware," in ICIP, 2007.

[35] I. Ernst and H. Hirschmüller, "Mutual information based semi-global stereo matching on the gpu," in $I S V C, 2008$.

[36] O. Rahnama, A. Makarov, and P. H. S. Torr, "Real-time depth processing for embedded platforms," in Proceedings of the SPIE, 2017.

[37] D. Hernandez-Juarez, A. Chacón, A. Espinosa, D. Vázquez, J. C. Moure, and A. M. López, "Embedded real-time stereo estimation via semi-global matching on the gpu," Procedia Computer Science, 2016. 